\title{
Novel Polarization Beam Splitter with High Fabrication Tolerance
}

\author{
Nicolás Abadía ${ }^{1,3}$, Xiangyang Dai ${ }^{2}$, Qiaoyin $\mathrm{Lu}^{2}$, Wei-Hua Guo², Md. Ghulam Saber ${ }^{3}$, David V. Plant ${ }^{3}$, and \\ John F. Donegan ${ }^{1}$ \\ ${ }^{1}$ Semiconductor Photonics Group, School of Physics and CRANN, Trinity College, Dublin 2, Ireland \\ ${ }^{2}$ Wuhan National Laboratory for Optoelectronics, Huazhong University of Science and Technology, Wuhan 430074, China \\ ${ }^{3}$ Department of Electrical and Computer Engineering, McGill University, Montreal, QC, H3A OE9, Canada \\ nicolas.abadiacalvo@mcgill.ca
}

\begin{abstract}
A highly fabrication tolerant polarization beam splitter is presented. The fabrication tolerances are relaxed by adjusting two voltages. Experiments show on-chip losses of $3.5 \mathrm{~dB}$ and extinction ratio of $15 \mathrm{~dB}$ at C-band.C 2018 The Author(s)

OCIS codes: (130.0250) Optoelectronics; (130.3120) Integrated optics devices; (130.5440) Polarization-selective devices
\end{abstract}

\section{Introduction and context}

Soon, the information in the Internet will reach the zettabyte level [1]. To deliver all this information, an upgrade to the optical links that carry this information is needed. One possible solution is to use coherent systems that have a good spectral efficiency. This kind of link is different from the optical links that use aggressive baud rates.

Among the coherent systems one possible solution is to use Dual-Polarization Quadrature-Phase Shift Keying (DP-QPSK) [2]. In such a link, one of the main components is the Polarization Beam Splitter (PBS). Such a device can separate a TE mode from a TM mode.

There are many PBS devices in both the silicon photonic platform [3-6] and in the III-V platform [7-10]. All of them have in common that they have tight fabrication tolerances. There are several designs that try to overcome such tolerances like the one presented in [9] that uses a Periodic Layer Structure of $\mathrm{Si} / \mathrm{SiO}_{2}$ or the ones [10-11] that uses thermal tuning. One of the main disadvantages of using the PLS is that it adds complexity to the fabrication. On the other hand, as demonstrated in [12] the thermal tuning has a difficult adjustment process that requires many iterative steps. This disadvantage was solved by the work proposed in [12]. By exploiting the Pockels effect in InP multiple quantum wells, the PBS function is achieved providing an easy adjustment. Nevertheless, a requirement of that design is that both phase shifters must be perpendicular to each other to have a proper crystal orientation required by the exploited effect. This leads to a big footprint around $1.5 \mathrm{~mm} \times 2.5 \mathrm{~mm}$.

In this work, we propose a novel PBS that is based on a symmetric 1x2 Mach-Zehnder interferometer (MZI) and exploits the plasma dispersion effect in forward bias and the Pockels effect in InP in reverse bias. The experimental results of the fabricated device show that an insertion loss of around $3.5 \mathrm{~dB}$ and a polarization extinction ratio better than $15 \mathrm{~dB}$ can be achieved at a wavelength of $1550 \mathrm{~nm}$. The device can operate over the entire C-band (1530-1565 $\mathrm{nm})$.

\section{Principle of operation}

The device is composed of a vertical p-i-n structure in an InP platform. If the p-i-n structure is in forward bias, the injected carriers change the refractive index of the material. This affects both the TE and the TM modes [13] in a similar way $\Delta \mathrm{n}_{\text {eff,TE }}\left(\mathrm{V}_{\text {forward }}\right) \approx \Delta_{\text {neff,TM }}\left(\mathrm{V}_{\text {forward }}\right)$, where $\Delta_{\text {neff,TE }}\left(\Delta_{\text {neff,TM }}\right)$ is the change in the effective index of the TE (TM) mode upon application of the forward bias voltage $V_{\text {forward }}$. This is the plasma dispersion effect.

In a different way, when a reverse bias voltage $V_{\text {reverse }}$ is applied to the $p$-i-n structure the Pockels effect changes the refractive index of the material. This effect affects only the TE mode [14]. Consequently, the change in the effective index of the TE mode is given by $\Delta \mathrm{n}_{\text {eff,TE}}\left(\mathrm{V}_{\text {reverse }}\right)$.

The structure of the proposed PBS consists of a symmetric 1x2 MZI as depicted in Fig.1 (a). The phase shifters of the structure are formed by vertical p-i-n structures. Different interference conditions of the $1 \times 2$ MZI are used to separate the TE from the TM mode. For this, one phase shifter is driven in forward bias and the other is driven in reverse bias. Since the plasma dispersion effect in forward bias affects both TE and TM and the Pockels effect in the phase shifter in reverse bias affects only the TE mode. Consequently, the TE and the TM modes have different interference conditions and by selecting $\mathrm{V}_{\text {forward }}$ and $\mathrm{V}_{\text {reverse }}$ the TE appears in the cross output and the TM appears in the bar output. Hence, producing the splitting function. To produce the PBS function the voltage $\mathrm{V}_{\text {forward }}$ and $\mathrm{V}_{\text {reverse }}$ need to be adjusted. To adjust $V_{\text {forward, }}$, first, a TM mode needs to be injected and $V_{\text {forward }}$ scanned. There will be a particular $\mathrm{V}_{\text {fordward }}$ at which the TM mode will exit the bar output (TM). $\mathrm{V}_{\text {forward }}$ must be set to that voltage. Once $\mathrm{V}_{\text {forward }}$ is set it is necessary to set $\mathrm{V}_{\text {reverse. }}$. For this, a TE mode is injected and the $\mathrm{V}_{\text {reverse }}$ is scanned. There will be a particular voltage in which the mode will exit the cross output (TE). The voltage $\mathrm{V}_{\text {reverse }}$ must be set to that voltage. In these two steps the splitting function is set and consequently the PBS function performed. 

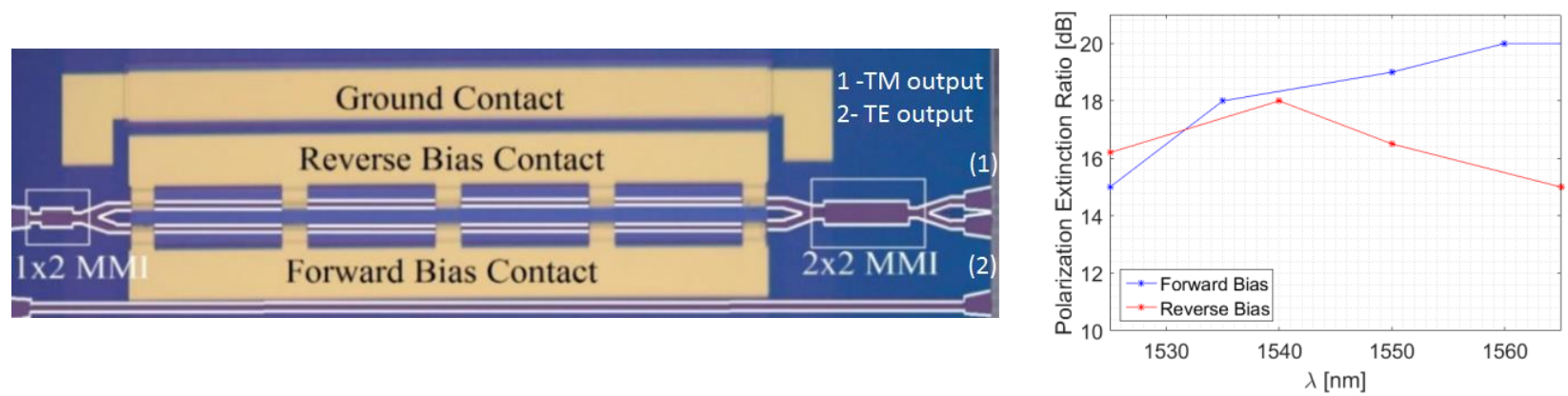

Figure 1: (a) Fabricated PBS with a straight waveguide in one side to measure the on-chip losses, (b) Measured polarization extinction ratio (PER) versus wavelength in the C-band

\section{Experimental demonstration}

The PBS was fabricated in order to demonstrate this proof-of-concept device. The measured polarization extinction ratio (PER) of the PBS in all the C-band $(1530-1565 \mathrm{~nm})$ is presented in Fig. 1 (a). As can be seen from Fig. 1 (b) the PBS works with a PER better than $15 \mathrm{~dB}$. The fabrication tolerance of our device is eased compared with those in the literature where variation in waveguide dimensions, thickness of the layers, etc. can be accommodated through small changes to both the forward and reverse bias conditions in the PBS. The proposed PBS has a better PER than the work presented in $[7,9,11,15]$. The work presented by Fraunhofer group in [10] has a better performance, nevertheless, to overcome the fabrication tolerances the device uses thermal tuning, which has a difficult calibration. This issue is solved by the work presented in [12]. This work can also be adjusted in two steps like the work presented here, nevertheless since the quantum confined Stark effect is used in quantum wells the device is wavelength dependent and consequently has a difficult adjustment across the C-band. We will also present the behavior of the PBS with the operational temperature and the fabrication tolerances.

\section{Conclusion}

We have demonstrated the proof-of-concept of a PBS which is fabrication tolerant by means of adjusting two voltages. Such a device exploits the plasma dispersion effect and the Pockels effect in a forward and reverse bias vertical pins structure in the arms of a symmetric 1x2 MZI. The experimental results shows that the PBS offer a better PER than $15 \mathrm{~dB}$ and an on-chip loss below $3.5 \mathrm{~dB}$ around $1550 \mathrm{~nm}$.

\section{References}

[1] CISCO, "The Zettabyte Era: Trends and Analysis," Cisco, no. May 2015, pp. 1-29, 2015.

[2] Q. Lu, et. al., "Optical Receiver," US 2013/0077980 A1, 2013.

[3] T. K. Liang, et. al., "Integrated polarization beam splitter in high index contrast silicon-on-insulator waveguides," IEEE Photonics Technol. Lett., vol. 17, no. 2, pp. 393-395, 2005.

[4] Y. Ding, et. al., "Wideband polarization splitter and rotator with large fabrication tolerance and simple fabrication process.," Opt. Lett., vol. 38 , no. 8, pp. 1227-9, 2013.

[5] X. Guan, et. al., "Ultracompact and broadband polarization beam splitter utilizing the evanescent coupling between a hybrid plasmonic waveguide and a silicon nanowire.," Opt. Lett., vol. 38, no. 16, pp. 3005-8, 2013.

[6] L. M. Line, et. al., “A Branch-Type TE / TM Wave Splitter Using a Light-Guiding Metal Line,” Lightwave, vol. 25, no. 3, pp. 922-928, 2007.

[7] F. H. Groen, et. al., "Mach-Zehnder Interferometer Polarization Splitter in InGaAsP/InP," IEEE Photonics Technol. Lett., vol. 6, no. 3, pp. $402-405,1994$.

[8] X. Guan, et. al., "Extremely small polarization beam splitter based on a multimode interference coupler with a silicon hybrid plasmonic waveguide," Opt. Lett., vol. 39, no. 2, pp. 259-62, 2014.

[9] D. Pérez-Galacho et. al., "Integrated polarization beam splitter with relaxed fabrication tolerances," Opt. Express, vol. 21, no. 12, pp. 14146$14151,2013$.

[10] M. F. Baier, et. al., "Polarization beam splitter building block for InP based generic photonic integrated circuits," Conf. Proc. - Int. Conf. Indium Phosphide Relat. Mater., pp. 1-2, 2014.

[11] M. Felicetti, et. al., "MMI-based polarization beam splitter/combiner for InP photonic integrated circuits," in Advanced Photonics 2015, 2015, p. IM2A.5.

[12] K. Wantabe, et. al., "Easy adjustment structure and method for realizing InP based polarization beam splitter via Pockels effect dependence on crystal orientation,” Jpn. J. Appl. Phys., vol. 55, no. 55, 2016.

[13] B. R. Bennett, et. al., "Carrier-Induced Change in Refractive Index of InP, GaAs, and InGaAsP," IEEE J. Quantum Electron., vol. 26, no. 1, pp. 113-122, 1990 .

[14] G. Hagn, "Electro-Optic Effects and Their Application in Indium Phosphide Waveguide Devices for Fibre Optic Access by Electro-optic," 1971.

[15] L. M. Augustin et. al., “A single etch-step fabrication-tolerant polarization splitter,” J. Light. Technol., vol. 25, no. 3, pp. 740-746, 2007.

[16] N. Abadía, et. al., "Highly fabrication tolerant InP based polarization beam splitter based on p-i-n structure," Opt. Express 25, 10070-10077 (2017) 